\title{
Virtual Microscopy in Undergraduate Pathology
} Education

\author{
An early transformative experience in clinical reasoning
}

\author{
Ritu Lakhtakia
}

\begin{abstract}
Objectives: Whole-slide imaging and virtual microscopy (VM) have revolutionised teaching, diagnosis and research in histopathology. This study aimed to establish the feasibility of achieving early integration of clinical reasoning with undergraduate pathology teaching on a VM platform and to determine its student-centricity through student feedback. Methods: This study was conducted at the Mohammed Bin Rashid University of Medicine and Health Sciences, Dubai, United Arab Emirates, between August and December 2017. A total of 38 VM-centred clinical cases were introduced to 49 students in an integrated undergraduate medical curriculum. The cases were aligned to curricular objectives, reinforced the pathologic basis of disease with critical thinking and were delivered across 15 interactive small-group sessions. A simulated cross-disciplinary integration and judicious choice of pertinent diagnostic investigations were linked to principles of management. Feedback was obtained through a mixed-methods approach. Results: User-friendliness, gradual learning curve of VM and annotation-capacity were scored as 4-5 (on a Likert scale of $1-5$ ) by $91.84 \%, 87.76 \%$ and $83.67 \%$ of the participants, respectively. Most students agreed that the content matched the stage of learning $(81.63 \%)$, theme of the week $(91.84 \%)$ and development of a strong clinical foundation (77.55\%). Integration (85.71\%) and clinico-pathological correlation (83.67\%) were the strengths of this educational effort. High student attendance $(\sim 100 \%)$ and improved assessment scores on critical thinking $(80 \%)$ were observed. Software lacunae included frequent logouts and lack of note-taking tools. Easy access was a significant student-centric advantage. Conclusion: A VM-centred approach with a clinico-pathological correlation has been successfully introduced to inculcate integrated learning. Using the pathologic basis of disease as a fulcrum and critical reasoning as an anchor, a digitally-enabled generation of medical students have embraced this educational tool for tutor-guided, student-centred learning.
\end{abstract}

Keywords: Digital Technology; Pathology; Microscopy; Medical Education; United Arab Emirates.

\section{Advances in KNOWLEdGe \\ Digital pathology is an enabler of integrated undergraduate medical education, blending clinical presentations with morphologic evidence of diseases to reinforce its pathophysiologic basis. \\ Judicious use of virtual microscopy (VM) in health profession-related education is invaluable in preparing future health professionals. Electronic tools are vital to student-centred learning in contemporary medical education.}

\section{Application to Patient Care}

$V M$ is increasingly being adopted in diagnostic practices, revolutionising modern histopathology laboratories.

It is appropriate that future doctors are sensitised to its manifold applications, while student-centred learning in today's digital generation is enhanced.

$\mathrm{T}$ He teaching of Diseased CONDitions IN undergraduate medical education has been dominated for more than two centuries by a macroscopic display of specimens mounted in pathology museums and microscopic cellular changes on glass slides. ${ }^{1}$ Over the last quarter-century, museums have reinvented learning through audiovisual compositions and tours. ${ }^{2}$ The $21^{\text {st }}$ century witnessed a transformation of the microscopic demonstration of diseases through the marriage of digital image capture (whole-slide imaging [WSI]) with software capabilities (virtual microscopy [VM]). This has resulted in a revolutionary transition in learning, liberating students from laboratory bench- top microscopes for conventional light microscopy (CLM). ${ }^{3,4}$ Pathology instructors have utilised this opportunity to design innovative teaching modules, while student-millennials have discovered ease and interest in tapping into this platform 'on the go', through computers and hand-held computing devices. ${ }^{5}$

The vertical integration of undergraduate learning with postgraduate training and subsequent entry into physician practice or academic career paths demands a nuanced, stage-specific exposure to modern technology. Digital microscopy has provided an opportunity for a second opinion on challenging diagnoses through remote consultation; several centres have shifted completely to virtual 
histopathology. ${ }^{6}$ Quality control programmes have increasingly transited to virtual slides to maintain speciality accreditation norms in histopathology. Sound underpinnings of microscopic evidence of diseases prepare physicians, irrespective of their chosen speciality, to make more informed decisions through multidisciplinary team meetings. Thus, it makes sense to embrace technology-enhanced microscopy and blend it with clinical emphasis to prepare future doctors.

This study was carried out in the newly established College of Medicine (CoM) at Mohammed Bin Rashid University of Medicine and Health Sciences (MBRU), Dubai Healthcare City, United Arab Emirates, which has an accredited, integrated medical curriculum with a student-centred, tutorenabled, educational philosophy. Digital learning and electronic assessment underpin the curricular experience. The six-year MBBS programme has nine programme learning outcomes (PLOs) to graduate a safe and competent doctor who practices evidence-based medicine with ethical attributes and a knowledge of modern healthcare systems. Good communication, advocacy and teamwork are essential hallmarks of this future physician with a strong capacity for self-directed learning. In the first pre-clinical year, students are introduced to structure and function, essentials of medical practice, bioethics, epidemiology and biostatistics. The second and thirdyear curricula integrate clinical manifestations and principles of management with disease morphology and its pathophysiologic basis.

This study was undertaken within a four-credit General Pathology course in the first semester of year two. The course introduces disease mechanisms and manifestation and specifically aligns with the PLOs in terms of knowledge and competency with clinicopathological correlation, communication, advocacy, peer education and self-directed learning. There is a spiral continuum with preceding basic sciences and succeeding clinical, stepwise learning. The emphasis is on clinical case-based teaching and early clinical-skills orientation in tandem, through simulations and hospital experience. Thus, this study was based on the constructivist theory of learning and aimed to enhance the spiral nature of the medical curriculum.

This study on digital pathology and VM as an educational intervention aimed to: a) establish the feasibility of achieving early integration of clinical reasoning with pathology teaching on a virtual microscopy platform and b) determine the studentcentricity of this teaching modality through student feedback. The objectives, design, delivery, student experience and assessment on this e-learning platform were analysed in this study. The likely impact of this innovative educational intervention is early integration of clinical reasoning with the fundamentals of disease and digital empowerment of $21^{\text {st }}$-century medical practitioners. The intent is to establish a continuum of undergraduate-postgraduate medical education.

\section{Methods}

An educational innovation of e-learning through VM was established at the CoM, MBRU, Dubai Healthcare City, United Arab Emirates. This is a new medical school that had its first cohort intake in 2016. The sixyear MBBS programme has three pre-clinical years followed by three clinical years. The first cohort intake had 54 students. A total of 49 students who progressed to year two participated in this study between August and December 2017. Students were introduced to VM through integrated clinical case-based tutorials in the General Pathology course which runs in the first semester of year two.

A web-based platform, Philips PathXL Tutor software (Cirdan PathXL Tutor, Lisburn, Ireland), was utilised for this educational delivery, accessed through an institutional subscription. It provided a whole scanned microscopic slide inventory comprising histology and histopathology slides that illustrated a range of common disease processes across organ systems. Dynamic VM viewing was accessed from the cloud of the software provider. The software tools enabled clinical case content to be built by tutors, by combining text and static pictures (clinical, radiology, electrophysiologic, macroscopic disease, multimedia, tables, etc.) and linking them with dynamic VM. Tutor training and student orientation formed part of the initiation process. Initial hurdles in software manipulation and annotation were reported to the website support team, who responded with software update solutions. This enabled a smooth and eventfree educational delivery.

VM-centred clinical case teaching was introduced in two-hour practical sessions within the General Pathology course. The cases illustrated the week's teaching theme and objectives such as cell injury, inflammation, oedema and thrombosis through fatty change, acute appendicitis, pulmonary oedema and thrombo-embolism, respectively. One case example of the 25 clinical scenarios taught during the semester is illustrated in Figure 1. Additional images and multimedia (e.g. radiology, electrocardiography, haematology, microbiology reports, etc.) were included 


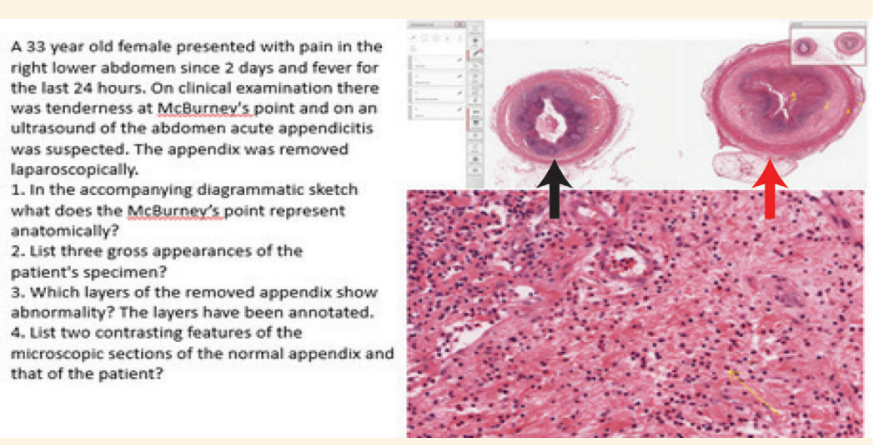

Figure 1: Composite illustration of a VM-centred prototype case (1 of 25 clinical scenarios) showing acute appendicitis. A clinical vignette integrates the anatomical basis of location of pain and the gross appearance of the operated appendix. Microscopic comparison of a normal (blue arrow) and diseased (red arrow) appendix at low magnification (upper right) and transmural neutrophilic infiltration at a higher magnification is possible.

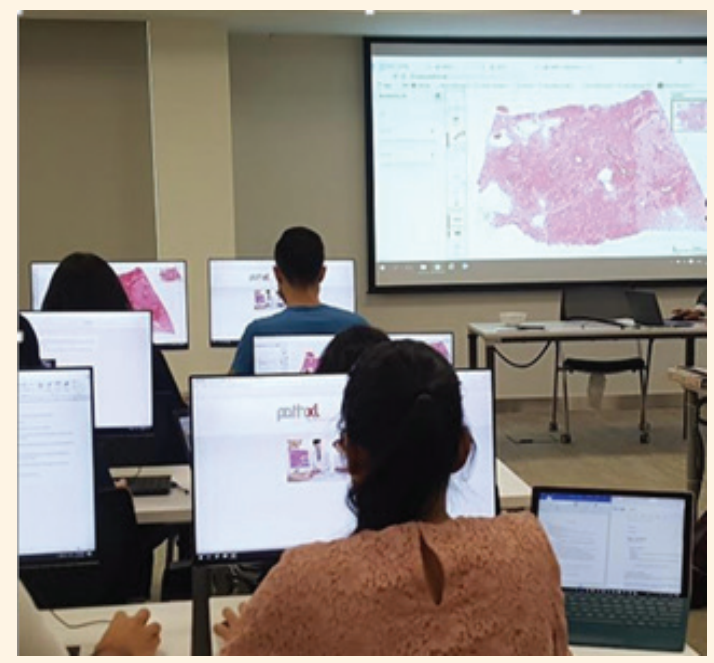

Figure 2: An interactive virtual microscopy session in progress in the computer laboratory at the Mohammed Bin Rashid University of Medicine and Health Sciences, Dubai, United Arab Emirates. Students dynamically manipulate the virtual microscope and discuss the questions and annotations with the tutor.

as appropriate. The clinical cases were designed inhouse to inculcate an early clinico-pathophysiologicmorphologic integration with the resultant ability to optimise the choice of investigation and rationalise diagnosis and management. Whole-slide images were uploaded to the case either alone or with normal histology slides to contrast normal with abnormal (e.g. normal myocardium with ischaemic necrosis in infarction) or features of two disease processes (e.g. lobar pneumonia versus bronchopneumonia). Slides were annotated to allow students to identify and discuss disease changes with the tutor and peers. Clinical complexity was appropriate to the stage of learning and integrated with the students' parallel learning of clinical skills and other diagnostic disciplines such as radiology, haematology, biochemistry, microbiology, etc.

A total of 15 interactive teaching sessions lasting two hours each, in a small group format, were conducted in a well-equipped computer laboratory [Figure 2]. Students had access to prepared cases on the website 'on the go' through their laptops, ahead of the formal scheduled discussion. The tutor projected the webpage live while students conducted the discussion. Tutor and student-annotated areas on VM were addressed. Case content was probed through interactive questions directed towards clinicopathological correlations, critical reasoning and logical thinking, and focused on identifying gaps in knowledge and comprehension. Follow-up enquiries from students, individually or through forums, closed the loop.

Student feedback was obtained at mid-semester through anonymous tutor-designed surveys and at end-semester through formal university-enabled electronic feedback on the courses. The surveys elicited the students' experience with VM (four questions) and content (five questions) through a mixed-methods approach. Quantitative responses on satisfaction with the VM experience on a four-item questionnaire were measured on a 5 -point Likert scale where $1=$ lowest and 5 highest. A five-item questionnaire on case content was scored from 1-3 as 'agree, 'partly agree' and 'disagree', respectively [Figures 3 and 4]. Qualitative responses sought anonymous, open-ended comments addressing lacunae and positive learning experiences for the improvement of the module and reinforcement of strengths, respectively.

Tutor feedback entailed inputs regarding courseappropriate case creation; learning curve on information technology (IT) tools including expertise and time management, navigation and ease of teaching on the web-based programme, mutual satisfaction between teacher and student in handling the oneon-one computer sessions and software support and troubleshooting by the website maintenance team.

An objective-structured practical examination (OSPE) was conducted through the Philips Tutor 


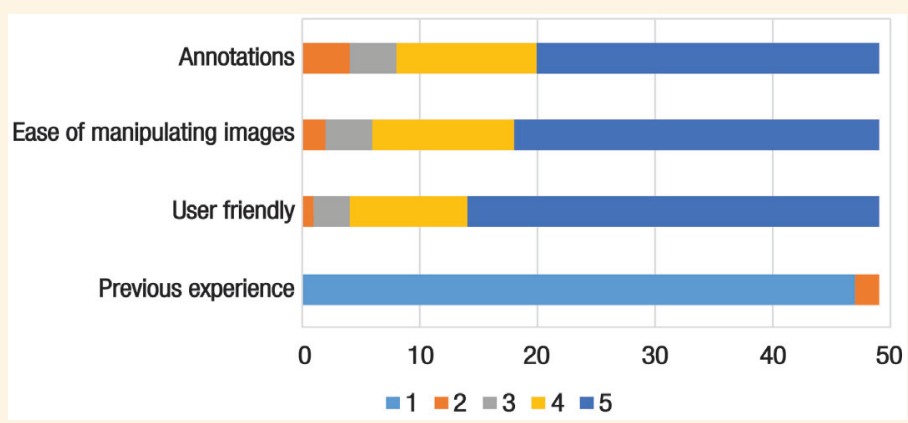

Figure 3: Quantitative responses of students' experience with virtual microscopy on a Likert scale of 1-5 where 1 is lowest and 5 is highest $(\mathrm{N}=49)$.

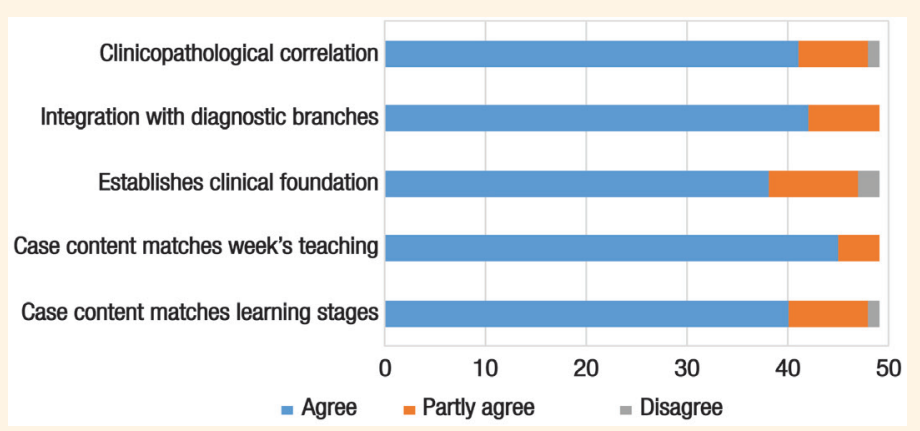

Figure 4: Student feedback on case content during a virtual microscopy course scaled quantitatively as 'agree, 'partly agree' and 'disagree' $(\mathrm{N}=49)$.

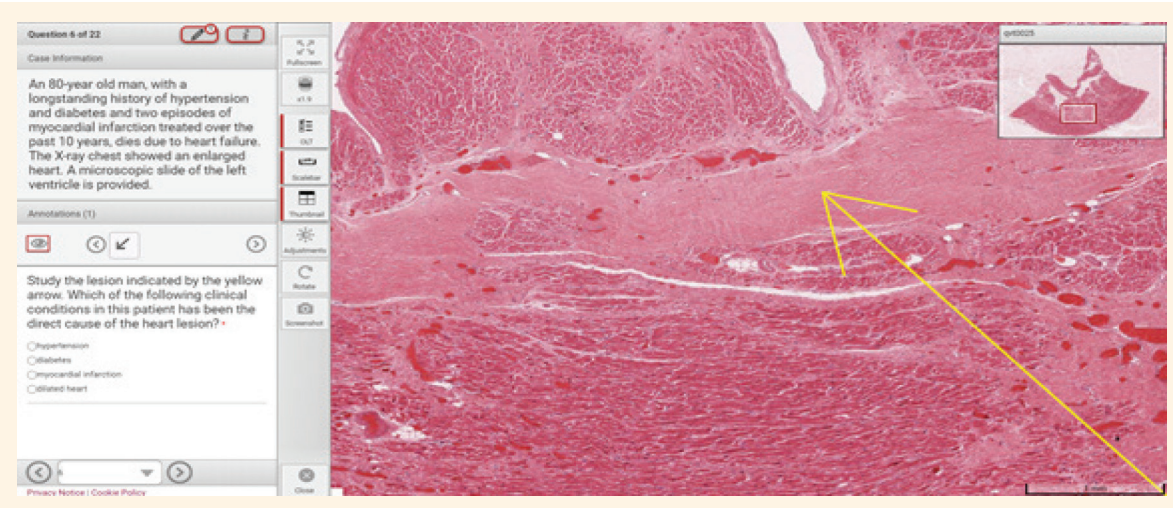

Figure 5: Example of the virtual microscopy software output showing a question that integrates clinical reasoning with disease morphology.

software's Online Testing and examination generation module (OLT) portal. It was weighted at $15 \%$ of the course's semester assessment. Students were provided formative guidance in the e-assessment of skills and in the question format. The skill domain was tested through OSPE stations that mirrored the teaching. Analysis of clinical vignettes centred on VM explored students' identification of disease morphology, for example, granulomas with clinicopathologic correlation and pathophysiologic interpretation of disease patterns. The format included VM slide-based annotations, multiple choice questions (MCQs) and short answers. A sample question is illustrated in Figure 5. The emphasis was not on mastery over histologic diagnosis, but on stage-appropriate knowledge of pathologic change that explains a clinical or radiologic manifestation or functional alteration due to disease.

An IRB exempt review was approved for this educational research: reference MBRU-IRB-2019-014. Informed consent was obtained from the students.

\section{Results}

For user friendliness, 45 out of 49 students (91.84\%) scored ease of software-use between 4-5. Specific suggestions for improvement were related to frustration with the 'time-out' mode, which required repeated log-in. The inability to write 'notes' on the 
same webpage while class discussions were ongoing was a further challenge. The capacity to manipulate VM images showed a gradual learning curve; 43 students $(87.76 \%)$ scored this attribute as a 4 or 5 . A part of the learning adaptation was the gradual recognition of cellular patterns from normal to abnormal. Annotation usage by students earned a score of 4-5 by 41 students (83.67\%). The interactive capacity of this tool, especially in out-of-class feedback, was especially useful, demonstrating self-directed learning [Figure 3].

This course was the students' first introduction to disease concepts and clinico-pathological correlation. A total of 40 out of 49 students (81.63\%) agreed that the content matched the stage of learning. Furthermore, 45 (91.84\%) students agreed that there was a correlation of practical cases to the didactic theme of the week. A total of 38 students (77.55\%) agreed that the course establishes a clinical foundation. In qualitative feedback, students expressed their initial struggle with clinical reasoning and a self-perceived gradual growth in capacity as they reached the end of the course, which was specifically attributed to case content. They identified frequent cross-integration with clinical skill learning in a simulated setting within the same semester. In addition, 42 students (85.71\%) agreed that the course cross-integrates pathology cases with other diagnostic modalities. The feedback acknowledged developing specific links with haematology, biochemistry and microbiology and critical learning of laboratory investigations in specific diseases. Students made particular mention of achieving vertical integration of disease identification in pathology with previous sensitisation to imaging in anatomy. Clinico-pathological correlation appeared to be a strength of this teaching format, with 41 students (83.67\%) agreeing that there was development of logical analysis of a clinical presentation. Student engagement was demonstrated by a near-100\% attendance across 15 face-to-face sessions [Figure 4].

Content design, developed in-house, was intellectually stimulating, resulting in a robust synthesis with the course's learning objectives. At each step, clinical reasoning, as the ultimate goal of pathology teaching, remained the guiding principle. Creation of case scenarios, familiarisation with software and IT troubleshooting was demanding. The most rewarding aspect was the interactive delivery and steady improvement in the students' analytical abilities over the period of study. The software assessment format, which allowed for the complex assembly of text, VM, annotations and static images, was effective in creating items that tested disease recognition and critical thinking.

The mean OSPE score of $82.14 \%$ (range 60-100\%) was similar to the average of the final semester theory examination. In the OSPE, students performed best on questions that integrated the analysis of symptoms, signs and radiology (80\%), with a lower average on functional correlations with organ disease (75\%), reflective of the early stage of learning. Assessment, in turn, provided an opportunity for formative feedback-individually and collectively-to address lacunae in fundamental disease concepts.

\section{Discussion}

Digital technology is ubiquitous and is an essential fact of human existence today whether in a social or professional arena. In the early $20^{\text {th }}$ century, CLM display, at first through analogue and later through digital video cameras, led to the evolution of telemedicine as a means of distance education. It was utilised by diagnostic disciplines such as radiology and anatomic pathology to enable second-opinion consultations by experts or for diagnostic coverage to remote areas. ${ }^{7}$ However, in educational terms, telepathology restricted display and manipulation to the instructor-operator. This prevented the exploratory learning that determines knowledge-skill integration. Pioneering efforts were made to explore replacing CLM with VM in the early $21^{\text {st }}$ century. ${ }^{8,9}$ Over the subsequent years, it became clear that the next logical step would be its application to medical teaching and diagnostic practice with the advancement of resolution in WSI. ${ }^{3,4}$ Today, it has become an enabling force in pathology education in many medical schools across the world, while efforts for standardising its usage in diagnostic practice are ongoing. ${ }^{10,11}$ Multicentric validation studies in recent times have provided an impetus to its acceptance, both in routine diagnostic reporting and for off-site frozen-section reporting. ${ }^{12,13}$ Virtual microscopy will play a vital role in the sharing of whole-slide images for research access across the scientific community as this facility expands, bridging opportunities across developing and developed nations. ${ }^{14}$

In pathology education, WSI and VM have overcome several shortcomings of the traditional microscope: (1) the need for producing multiple glass slides from limited tissues for individual viewing by a large number of students; (2) the limitation of the number of slides in cytology preparation; (3) replenishment of slides for faded stains or broken/lost slides; (4) individual supervision during the learning process; and (5) laboratory-bound access to learning. There are, in addition, two potent arguments for VM in undergraduate medical education: the ease with which a generation born to the digital age accepts and enjoys it and the robust shift from tutor-directed to 
student-centred learning, as it is a 'take-anywhere', 'use-anytime' tool. The ergonomic ease of VM over CLM can hardly be overemphasised.

In the setting of a new medical school, the use of VM $a b$ initio provided all these advantages. The students in this study cohort had limited parallel experience on microscope hardware usage in two practicals each for the Microbiology and Haematology courses delivered in the same semester. However, students showed rapid engagement with the webbased platform, which provided ease of access and content exploration. Microscopic slide resolution was excellent and matched glass slide clarity during teaching and learning. While the students have excellent digital skills growing up as a digitally enabled generation, VM was virgin territory. Once mastered, the ease of image manipulation provided a gaming effect to stimulate student learning. An interesting off-shoot was the high rate of student attendance, validating enthusiasm for this simulated learning platform and its perceived benefits.

Marchevsky et al. reported the switchover to case-based teaching of pulmonary pathophysiology on digitised images aided by text and audio recordings in the University of California-Los Angeles, guided by a pathologist-pulmonologist tutor team. ${ }^{8}$ Observation over a four-year period reported stimulated student interest upping attendance from $30-40 \%$ to $100 \%$ and progressive student satisfaction. ${ }^{8}$ In the current study cohort, student attendance had been unprecedented, validating student interest. Steinberg et al's study illustrating cytopathology applications in clinical practice to second year students, using 10 electronic, interactive modules and evaluation by MCQs showed demonstrable student satisfaction. ${ }^{9}$ WSI and VM adaptive tutorials were used in a randomised crossover trial as tools to introduce cytopathology to senior medical students at the University of New South Wales, who were previously naive to the subject matter. ${ }^{15}$

Physician assistants exposed to video-assisted microscopy showed significantly higher practical examination scores compared to five previous cohorts that used conventional microscopy. ${ }^{16}$ This is attributed largely to two processes. First, the discussions that go beyond textbook facts to real-life simulated cases and problem-solving capability that allows students to take ownership of the process; and second, the design of the assessment itself that stimulates critical thinking, which is a consequence of the students' approach to these sessions. ${ }^{6}$

At a new medical school, introduction of VM from the beginning, when creating an integrated curriculum and choosing a digital format of delivery, was a well- considered decision. The continuum of learning normal histology in tandem with histopathology on a dynamic image mode was perceived by students as an enriching experience. Students' interpretive capacity improved from their first exposure continually through the semester and dramatically in the following semesters, as they followed pathology learning in the organ systems (data not shown). Students had the freedom to explore and annotate images within the construct of clinical cases; the simulation provided an enriched, clinically relevant interpretation of disease while encouraging collaborative peer learning, as also reported by others. ${ }^{17}$

The focus on clinical reasoning, on the one hand, takes away the tedium of looking at static images of morphologic changes and learning by rote to 'spot' diseased cells and tissues. On the other hand, it avoids the ocular adaptation challenges to the physical microscope and its restricted availability, as it is confined to the educational site.

There is a strategic approach that graduate students take, from initially engaging in simple disease-based integrative learning to dealing with the complexities of presentation-based differential diagnosis in their third year with increasing clinical maturity. The foundation laid in year two transits to pathology teaching within six integrated organ-systems courses over the next three semesters (years two and three) of the curriculum. In this way, within and across the organ systems, spiral learning is achieved. A VMcentred approach enhances this learning journey and will lead students to the clerkship years, armed with a robust understanding of diseases.

VM has already entered the arena of graduate medical education in histopathology, haematology and dermatology, wherein large libraries of digitised slides, available via open access or through subscription, enable the mastery of a range of disease diagnoses beyond the confines of clinical training sites. $^{18,19}$ In a nation-wide survey in the USA across 52 histopathology residency programmes, $82 \%$ used web-based digital slide collections. ${ }^{18}$ In-training and certification examinations now use a VM platform. In addition, anatomic pathology and cytopathology quality assurance programmes have already adopted it to ensure safe diagnostic expertise..$^{20,21}$ Thus, the continuum of undergraduate-postgraduate learning of VM is now an established need. Continuing efforts and validation have brought VM into the realm of diagnostic histopathology with some centres having taken the leap to exclusive digital practice..$^{22,23}$

Medical practice, whatever the chosen specialty, rests on sound knowledge, skills and attitudes. Visual demonstrations of disease recognition, whether clinical 
or in diseased organs, assume multiple forms and the VM mode has brought another nuance to learning styles. Almost all hospital-based specialty practice entails multidisciplinary team (MDT) meetings to secure rational and comprehensive management decisions. Today, VM in one form or another, occupies centre-stage in an MDT's ultimate aim of making informed decisions on patient management. ${ }^{6}$ Irrespective of the chosen specialty, it will become a vital $\operatorname{cog}$ in the learning wheel of a competent medical professional when practicing safe medical care; hence, its use early in the educational process seems entirely appropriate.

This study was subject to two main limitations. First, the instructional strategy was implemented in the first cohort of students at a new medical school; hence, it lacks a comparison with a control group that underwent simultaneous conventional microscopy teaching. Second, the analyses can be improved by surveying the same cohort once they enter clinical clerkship, to reflect on the value of early clinical reasoning integrated with student-centred teaching of VM pathology.

\section{Conclusion}

Digital pathology and VM span the continuum of learning about disease mechanisms and appearances from undergraduate or postgraduate levels to continued medical education. The art of its usage relies on the careful construction of teaching content, to make it the backbone for clinical reasoning and critical thinking. This study demonstrates its successful implementation in integrated clinical teaching in a new medical school and establishes its utility through student satisfaction and assessment. Its usage finds vindication in the current COVID-19 pandemic when remote digital delivery has proven to be the saviour of education. VM is, today, an integral part of quality assurance, research and multidisciplinary meetings for patient management. Its introduction into diagnostic practice and specialised cross-consultations is a work in progress, subject to validation and regulation. The growing digital platform, integrated with advances in high-resolution images, holds the future of education, research and patient care.

\section{CONFLICT OF INTEREST}

The author declares no conflicts of interest.

\section{FUNDING}

No funding was received for this study.

\section{References}

1. Marreez YM, Willems LN, Wells MR. The role of medical museums in contemporary medical education. Anat Sci Educ 2010; 3:249-53. https://doi.org/10.1002/ase.168.

2. Nesi G, Santi R, Taddei GL. Art and the teaching of pathological anatomy at the University of Florence since the nineteenth century. Virchows Arch 2009; 455:15-19. https://doi.org/10.10 07/s00428-009-0794-y.

3. Dee FR. Virtual microscopy in pathology education. Hum Pathol 2009; 40:1112-21. https://doi.org/10.1016/j.humpath.2009.04.010.

4. Foster K. Medical education in the digital age: Digital whole slide imaging as a learning tool. J Pathol Inform 2010; 1:14. https://doi.org/10.4103/2153-3539.68331.

5. Saco A, Bombi JA, Garcia A, Ramírez J, Ordi J. Current status of whole-slide imaging in education. Pathobiology 2016; 83:79-88. https://doi.org/10.1159/000442391.

6. OrahM, RotimiO. TelepathologyinlowresourceAfricansettings. Front Public Health 2019; 7:264. https://doi.org/10.3389/fpu bh.2019.00264.

7. Weinstein RS, Graham AR, Richter LC, Barker GP, Krupinski EA, Lopez AM, et al. Overview of telepathology, virtual microscopy, and whole slide imaging: Prospects for the future. Hum Pathol 2009; 40:1057-69. https://doi.org/10.1016/j.humpath.2009.04.006.

8. Marchevsky AM, Relan A, Baillie S. Self-instructional "virtual pathology" laboratories using web-based technology enhance medical school teaching of pathology. Hum Pathol 2003; 34:423-9. https://doi.org/10.1016/s0046-8177(03)00089-3.

9. Steinberg DM, Chan TY, Freedman JA, Grimm LA, Ling L, Lehmann HP, et al. Teaching cytopathology to second-year medical students. An interactive, case-based approach. Acta Cytol 2002; 46:481-9. https://doi.org/10.1159/000326865.

10. Campbell WS, Hinrichs SH, Lele SM, Baker JJ, Lazenby AJ, Talmon GA, et al. Whole slide imaging diagnostic concordance with light microscopy for breast needle biopsies. Hum Pathol 2014; 45:1713-21. https://doi.org/10.1016/j.humpath.20 14.04.007.

11. Eccher A, Neil D, Ciangherotti A, Cima L, Boschiero L, Martignoni G, et al. Digital reporting of whole-slide images is safe and suitable for assessing organ quality in preimplantation renal biopsies. Hum Pathol 2016; 47:115-20. https://doi. org/10.1016/j.humpath.2015.09.012.

12. Baidoshvili A, Stathonikos N, Freling G, Bart J, 't Hart N, van der Laak J, et al. Validation of a whole-slide image-based teleconsultation network. Histopathology 2018; 73:777-83. https://doi.org/10.1111/his.13673.

13. Pradhan D, Monaco SE, Parwani AV, Ahmed I, Duboy J, Pantanowitz L. Evaluation of panoramic digital images using Panoptiq for frozen section diagnosis. J Pathol Inform 2016; 7:26. https://doi.org/10.4103/2153-3539.181770.

14. Lee LMJ, Goldman HM, Hortsch M. The virtual microscopy database-sharing digital microscope images for research and education. Anat Sci Educ 2018; 11:510-15. https://doi.org/10.10 02/ase.1774.

15. Van Es SL, Kumar RK, Pryor WM, Salisbury EL, Velan GM. Cytopathology whole slide images and adaptive tutorials for senior medical students: A randomized crossover trial. Diagn Pathol 2016; 11:1. https://doi.org/10.1186/s13000-016-0452-z.

16. McDaniel MJ, Russell GB, Crandall SJ. Innovative strategies for clinical microscopy instruction: Virtual versus light microscopy. J Physician Assist Educ 2018; 29:109-14. https://doi. org/10.1097/JPA.0000000000000198.

17. Braun MW, Kearns KD. Improved learning efficiency and increased student collaboration through use of virtual microscopy in the teaching of human pathology. Anat Sci Educ 2008; 1:240-6. https://doi.org/10.1002/ase.53. 
18. Vallangeon BD, Hawley JS, Sloane R, Bean SM. An assessment of pathology resident access to and use of technology: A nationwide survey. Arch Pathol Lab Med 2017; 141:431-6. https://doi.org/10.5858/arpa.2016-0228-OA.

19. Kent MN, Olsen TG, Feeser TA, Tesno KC,Moad JC, Conroy MP, et al. Diagnostic accuracy of virtual pathology vs traditional microscopy in a large dermatopathology study. JAMA Dermatol 2017; 153:1285-91. https://doi.org/10.1001/jamadermatol.2017.3284.

20. Ross J, Greaves J, Earls P, Shulruf B, Van Es SL. Digital vs traditional: Are diagnostic accuracy rates similar for glass slides vs whole slide images in a non-gynaecological external quality assurance setting? Cytopathology 2018; 29:326-34. https://doi. org/10.1111/cyt.12552.
21. Van Es SL, Greaves J, Gay S, Ross J, Holzhauser D, Badrick T. Constant quest for quality: Digital cytopathology. J Pathol Inform 2018; 9:13. https://doi.org/10.4103/jpi.jpi_6_18.

22. Goacher E, Randell R, Williams B, Treanor D. The diagnostic concordance of whole slide imaging and light microscopy: A systematic review. Arch Pathol Lab Med 2017; 141:151-61. https://doi.org/10.5858/arpa.2016-0025-RA.

23. Stathonikos N, Veta M, Huisman A, van Diest PJ. Going fully digital: Perspective of a Dutch academic pathology lab. J Pathol Inform 2013; 4:15. https://doi.org/10.4103/2153-3539.114206. 